\title{
Optofluidic tuning of photonic crystal band edge lasers
}

\author{
Bernal, Felipe; Christiansen, Mads Brøkner; Gersborg-Hansen, Morten; Kristensen, Anders
}

\section{Published in:}

Applied Physics Letters

Link to article, DOI:

$10.1063 / 1.2817610$

Publication date:

2007

Document Version

Publisher's PDF, also known as Version of record

Link back to DTU Orbit

Citation (APA):

Bernal, F., Christiansen, M. B., Gersborg-Hansen, M., \& Kristensen, A. (2007). Optofluidic tuning of photonic crystal band edge lasers. Applied Physics Letters, 91(22), 223503. https://doi.org/10.1063/1.2817610

\section{General rights}

Copyright and moral rights for the publications made accessible in the public portal are retained by the authors and/or other copyright owners and it is a condition of accessing publications that users recognise and abide by the legal requirements associated with these rights.

- Users may download and print one copy of any publication from the public portal for the purpose of private study or research.

- You may not further distribute the material or use it for any profit-making activity or commercial gain

- You may freely distribute the URL identifying the publication in the public portal

If you believe that this document breaches copyright please contact us providing details, and we will remove access to the work immediately and investigate your claim. 


\title{
Optofluidic tuning of photonic crystal band edge lasers
}

\author{
Felipe Bernal Arango, ${ }^{\text {a) }}$ Mads Brøkner Christiansen, \\ Morten Gersborg-Hansen, and Anders Kristensen ${ }^{\text {b) }}$ \\ MIC-Department of Micro and Nanotechnology, Nano DTU, Technical University of Denmark, \\ Building 345E, DK-2800 Kgs. Lyngby, Denmark
}

(Received 10 October 2007; accepted 5 November 2007; published online 26 November 2007)

\begin{abstract}
We demonstrate optofluidic tuning of polymer photonic crystal band edge lasers with an imposed rectangular symmetry. The emission wavelength depends on both lattice constant and cladding refractive index. The emission wavelength is shown to change $1 \mathrm{~nm}$ with a cladding refractive index change of $10^{-2}$. The rectangular symmetry modification alters the emission characteristics of the devices and the relative emission intensities along the symmetry axes depend on cladding refractive index, suggesting a sensor concept based on detection of intensity rather than wavelength. () 2007 American Institute of Physics. [DOI: 10.1063/1.2817610]
\end{abstract}

Optical means of detection in lab-on-a-chip systems, i.e., integrated analysis systems, are often advantageous. ${ }^{1,2}$ Through the integration of microfluidics and optics, the field of optofluidics has emerged, ${ }^{3,4}$ enabling reconfigurable optical devices, such as tunable optofluidic dye lasers, ${ }^{5,6}$ suitable for sensing applications. ${ }^{7}$ Photonic crystal $(\mathrm{PhC})$ structures $^{8}$ can be very sensitive to changes in the refractive index of their immediate surroundings, ${ }^{9}$ especially when chemical or biological analytes are immobilized on their surfaces, within their evanescent optical fields. ${ }^{10,11}$ Several $\mathrm{PhC}$ refractive index sensors made from semiconductor materials have been demonstrated, ${ }^{10-14}$ although good sensitivities have been obtained, they are expensive to fabricate, in particular, if optical gain is required.

Optical gain in the visible region, which is attractive for applications in chemistry and biology, ${ }^{1}$ can be obtained from semiconducting polymers or transparent polymers doped with organic dyes. Many polymers can be structured by nanoimprint lithography (NIL), enabling cheap parallel fabrication of large numbers of devices. Recently, a number of nanoimprinted photonic crystal band edge lasers ${ }^{15}$ (PhCBELs) have been realized. ${ }^{16-20}$

In this letter, we impose a rectangular cavity on triangular lattice polymer PhCBELs, thereby modifying the sixfold symmetry in order to reduce the number of emission directions from six to four. The feedback of the cavity is obtained as a combination of the distributed feedback of the $\mathrm{PhC}$ structure and reflections on the sidewalls of the imposed cavity. This causes two of the $\mathrm{PhC}$ modes to collapse into one mode emitting along the length of the rectangular device. Through optofluidic tuning of the devices, by changing the cladding refractive index, we investigate the influence on emission wavelength and intensities of emission along the axes of the rectangular cavity. The results reveal polymer PhCBELs as promising candidates for future application in low cost highly integrated sensor concepts.

A schematic drawing of the PhCBELs used can be seen in Figs. 1(a) and 1(b). The lasers are slab waveguides of the photodefinable polymer SU-8 (MicroChem) doped with the

\footnotetext{
${ }^{a)}$ Present address: Kavli Institute of Nanoscience, Delft University of Technology, Lorentzweg 1, 2628 CJ Delft, The Netherlands.

${ }^{b)}$ Author to whom correspondence should be addressed. Electronic mail: ak@mic.dtu.dk; URL: www.mic.dtu.dk/ak
}

organic laser dye Pyrromethene 597 (PM597) from Exciton. The footprint of the devices is $250 \mu \mathrm{m} \times 1 \mathrm{~mm}$ and the height of the waveguide $h$ is approximately $450 \mathrm{~nm}$. The photonic crystals, which generate the feedback enabling laser oscillations, are imprinted approximately $100 \mathrm{~nm}$ into the polymer surface. The PhCs are fabricated with triangular lattices of air holes with lattice constants $a$ varying from 364 to $424 \mathrm{~nm}$.

The band structure is calculated with a freely available Maxwell's equations eigenmode solver. ${ }^{21}$ The calculations are done in two dimension, where the indices in and around the holes are calculated as the effective refractive index of corresponding slab waveguides. ${ }^{22,23}$ The lasers are designed to emit at one of two TE-like zero group velocity points located in the $\Gamma$ and $M$ points around the second and fourth bands, respectively. ${ }^{8}$ Band structures are calculated individually for each device, typical frequencies for the two points are $(\Gamma, 0.745 c / a)$ and $(M, 0.654 c / a)$. By changing the lattice constant $a$, one of the two points is positioned in the gain spectrum of the dye $\left(\lambda_{\text {gain }} \approx\{532-650 \mathrm{~nm}\}\right)$. The lattice constants chosen are $364,384,404$, and $424 \mathrm{~nm}$, all with $r / a$ $=0.3$ where $r$ is the hole radius. The $a \leqslant 404 \mathrm{~nm}$ devices are
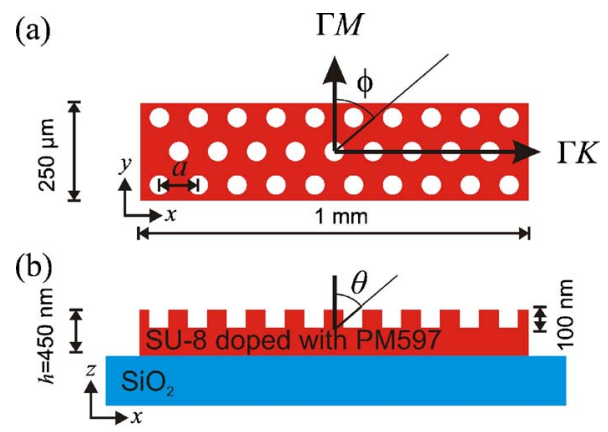

(c)

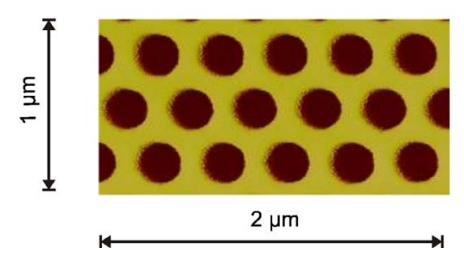

FIG. 1. (Color online) [(a) and (b)] Schematical top and side views of the fabricated photonic crystal band edge lasers. (c) AFM image of an imprinted laser. 


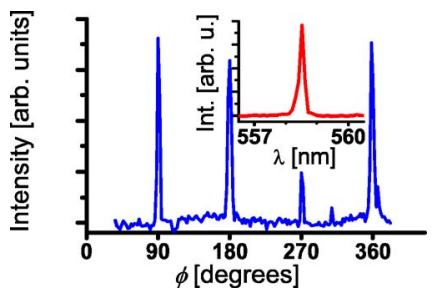

FIG. 2. (Color online) Intensity as a function of azimuthal angle $\phi$ for $a$ $=364 \mathrm{~nm}$ device of height $h=435 \mathrm{~nm}\left(\theta=70^{\circ}\right)$. The emission directions correspond to the symmetry axes of the imposed rectangular cavity of the device. The wavelength is constant and corresponds to $M$ point emission. The intensity differences of the peaks may be attributed to slight differences in pumping conditions as the laser is rotated. Inset shows a typical laser spectrum (linewidth around $0.15 \mathrm{~nm}$ ).

expected to emit from the $M$ point and the devices with $a$ $=424 \mathrm{~nm}$ are expected to emit out of plane from the $\Gamma$ point $\left(\theta=0^{\circ}\right)$. The chosen $M$ and $\Gamma$ points are located outside the light cone of the band structure.

Fabrication of the lasers is done by combined nanoimprint and photolithography, ${ }^{24}$ using an UV transparent $10 \mathrm{~cm}$ diameter imprint stamp with an integrated $\mathrm{Cr}$ shadow mask. The nanometer features are first replicated in the PM597 doped SU-8 film in a conventional NIL step, and the polymer film is subsequently crosslinked selectively by UV exposing through the shadow mask of the stamp. Finally, the reusable stamp is separated from the substrate and the unexposed SU-8 is dissolved. Process details can be found in Refs. 25 and 26. An atomic force microscopy (AFM) image of a section of the PhCs can be seen in Fig. 1(c).

The PhCBELs are optically pumped from above at $532 \mathrm{~nm}$ using a frequency doubled $\mathrm{Nd}$ doped yttrium aluminum garnet laser, $5 \mathrm{~ns}$ pulse duration. The emitted light is collected by an optical fiber connected to a fixed grating spectrometer (Ocean Optics HR2000) with a resolution of $0.15 \mathrm{~nm}$.

A typical laser spectrum of a PhCBEL with $a=364 \mathrm{~nm}$ is seen in the inset of Fig. $2\left(\phi=0^{\circ}, \theta=70^{\circ}\right)$. For the $a$ $=364 \mathrm{~nm}, a=384 \mathrm{~nm}$, and $a=404 \mathrm{~nm}$ devices, the wavelength and direction of emission correspond to $M$ point oscillations when measured from $\phi=0^{\circ}$ (see Table I). The spectral width is close to the resolution of the spectrometer. At high pump fluences, a secondary peak with a wavelength corresponding to the other band edge at the $M$ point appears. The emission of the $a=424 \mathrm{~nm}$ devices was vertically up, as expected, with three to four closely spaced narrow peaks. The wavelengths of the peaks correspond to different bands in the $\Gamma$ point (see Table I). There are a total of six bands at the $\Gamma$ point in the gain spectrum of the dye. Laser threshold pump pulse fluences from $\sim 1$ to $20 \mu \mathrm{J} / \mathrm{mm}^{2}$ were measured.

Figure 2 shows the $\phi$ dependence of the laser intensity at $\theta=70^{\circ}$ for $a=364 \mathrm{~nm}$ device. The wavelength is constant within $0.15 \mathrm{~nm}$ irrespective of the measurement angles. The

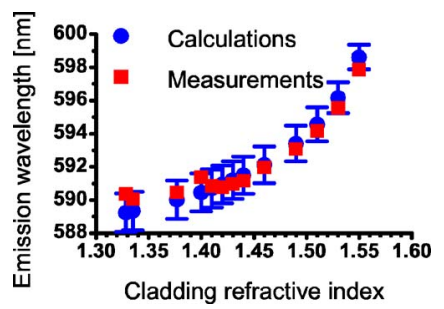

FIG. 3. (Color online) Optofluidic tuning. Measured (red squared) and calculated (blue circles) emission wavelengths for $a=384 \mathrm{~nm}$ device as a function of cladding refractive index $\left(h=435 \mathrm{~nm}, \phi=0^{\circ}\right.$, and $\left.\theta=62^{\circ}\right)$. The error bars reflect the uncertainty of the measured SU- 8 refractive index. Each point is calculated individually to take into account wavelength dispersion in the waveguide structure.

laser emission is primarily along the symmetry axes of the rectangular laser footprint. The signals at $\phi=0^{\circ}$ and $\phi=180^{\circ}$ are expected, since these correspond to $\Gamma M$ directions in the PhC. No emission is observed in the remaining four $\Gamma M$ directions. In the $\phi=90^{\circ}$ and $\phi=270^{\circ} \Gamma K$ directions, emission with a wavelength corresponding to the $M$ point is observed. This is attributed to coupling of $M$ point emission into waveguide modes defined by the rectangular imposed cavity. Another indication of cavity coupling is that the $\phi=0^{\circ}$ and $\phi=180^{\circ}$ emissions are maximal with a narrow angular distribution around the expected $\theta=62.3^{\circ}$, whereas the $\phi=90^{\circ}$ and $\phi=270^{\circ}$ emissions are maximal around $\theta=68^{\circ}$, closer to in-plane emission and with a broad angular distribution, as expected for end emission from a thin unstructured waveguide. The narrow $\theta$ distributions in the $\phi=0^{\circ}$ and $\phi=180^{\circ}$ directions are indications of few defects in the lattice. ${ }^{27}$

The feedback properties of the PhCs are tuned by cladding the lasers with fluids of different refractive indices. This causes the emission wavelength to change, as shown in Fig. 3 for an $a=384 \mathrm{~nm}$ device $\left(\phi=0^{\circ}, \theta=62^{\circ}\right)$. Between each measurement, the device is cleaned with methanol and dried using nitrogen. In the calculated data, the refractive indices of glass and SU-8 are assumed to be 1.46 and 1.5965 , respectively. The error bars in the model data indicate the uncertainty of the SU-8 refractive index. The SU-8 index was measured between 1.593 and 1.600 with a SCI Filmtek 4000 thin film measurement system. For cladding refractive indices close to that of SU-8, the laser emission might be expected to regain the symmetry of the PhC structures. However, when high index claddings were used, no laser emission was observed due to reduced $\mathrm{PhC}$ feedback.

The sensitivity of the lasers to refractive index changes of their surroundings make them promising candidates for use as optofluidic sensors. The spectrally narrow emission makes detection of small wavelength changes feasible. The slope of the curve in Fig. 3 is seen to be maximal around 1.55 , corresponding to a $1 \mathrm{~nm}$ change in emission wavelength per $10^{-2}$ change in refractive index. A refractive index

TABLE I. Measured $\lambda_{\text {meas }}$ and calculated $\lambda_{\text {calc }}$ wavelength values for lasers with different lattice constants $a(h=480 \mathrm{~nm})$. Calculations are done individually for each laser, to take account of dispersion in the effective refractive index calculations. The values of $\lambda_{\text {calc }}$ are predicted within $\pm 1 \mathrm{~nm}$ due to the uncertainty of the SU-8 refractive index. The uncertainty of $\lambda_{\text {meas }}$ is $0.15 \mathrm{~nm}$ due to limited spectrometer resolution.

\begin{tabular}{|c|c|c|c|c|}
\hline$a(\mathrm{~nm})$ & 364 & 384 & 404 & 424 \\
\hline$\lambda_{\text {calc }}(\mathrm{nm})$ & 560.2 & 589.9 & 619.3 & $564.1,565.0,{ }^{\mathrm{a}} 565.7,{ }^{\mathrm{a}} 566.6$ \\
\hline$\lambda_{\text {meas }}(\mathrm{nm})$ & 560.6 & 590.9 & 618.3 & $563.1,566.6,567.7$ \\
\hline
\end{tabular}

${ }^{\mathrm{a}}$ Twofold degenerate. 


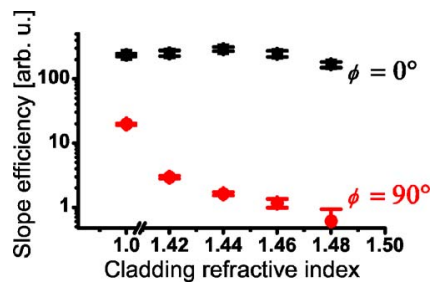

FIG. 4. (Color online) External slope efficiency $\left(\Delta I_{\text {out }} / \Delta I_{\text {pump }}\right)$ of the emission as a function of cladding refractive index when measured from $\phi=0^{\circ}$ (black squares) and $\phi=90^{\circ}$ (red circles) for the $a=384 \mathrm{~nm}$ device of Fig. 3. The error bars reflect the error of the fit to the measured data.

change of $10^{-3}$ can be easily detected and the sensitivity at lower refractive indices could be increased through design optimization, e.g., by using thinner polymer films.

If the surface of the lasers were functionalized to bind specific analytes as in Ref. 10, it could provide two advantages. The first is that the sensitivity could be increased substantially since the analyte would be concentrated near the device surface, within the evanescent optical field. Secondly, several devices prepared for the binding of different analytes could be positioned along the same microfluidic channel, enabling parallel multiplexed detection of chemicals or proteins.

Since the sensor is a laser by itself, it is easy to operate, as the pump light simply has to be absorbed by the laser dye. Further, a waveguide can be integrated for collecting the light, as in Ref. 26. These advantages simplify addressing individual devices if several sensors for different analytes are compactly integrated.

Figure 4 shows the external slope efficiency $\Delta I_{\text {out }} / \Delta I_{\text {pump }}$ of the $a=384 \mathrm{~nm}$ device of Fig. 3, as a function of cladding index, when the signal is measured from the $\phi=0^{\circ}$ and $\phi=90^{\circ}$ directions, respectively (intensity optimized with regard to $\theta$ ). The slope efficiency is approximately constant when measuring from the $\phi=0^{\circ}$ direction but it drops for increasing cladding refractive index when measuring from the $\phi=90^{\circ}$ direction, indicating coupling between the $\mathrm{PhC}$ and the rectangular imposed cavity. We attribute the decrease of slope efficiency at $\phi=90^{\circ}$ to a lower ability of the imposed cavity to guide the light, due to lower index contrast. This is supported by the fact that the thresholds for lasing in both directions are approximately constant irrespective of the cladding index, indicating that the total loss of the PhCBEL exhibits only small variations.

The results suggest a sensor concept utilizing the change in slope efficiency in the $\phi=90^{\circ}$ direction. For a given pump condition, the $\phi=90^{\circ}$ emission intensity depends on cladding refractive index, while the $\phi=0^{\circ}$ emission can be used as a reference. This enables detection based on intensity measurements rather than wavelength, possible by simple integration of photodiodes in the system, as in Ref. 7.

In conclusion, we demonstrate polymer photonic crystal band edge lasers with an imposed rectangular symmetry yielding an alteration of the emission directions, compared with the local symmetry directions of the $\mathrm{PhC}$ lattice. The lasers are fabricated in a parallel manner and are simple to use as optofluidic sensors. The emission wavelength is shown to depend on the refractive index of the cladding with a sensitivity of $1 \mathrm{~nm} / 10^{-2}$ refractive index unit. Furthermore, the slope efficiencies for emission along the two symmetry axes are shown to be different, suggesting a sensor concept where the relative intensity of the light in the two directions is measured, rather than the emission wavelength.

The partial support of the EC-funded project $\mathrm{NaPa}$ (Contract No. NMP4-CT-2003-500120) is gratefully acknowledged.

${ }^{1}$ E. Verpoorte, Lab Chip 3, 42N (2003).

${ }^{2}$ L. Lading, L. B. Nielsen, and T. Sevel, in Proceedings of IEEE Sensors 2002 (Institute of Electrical and Electronics Engineers, New York, 2002), Vol. 1, pp. 229-232.

${ }^{3}$ D. Psaltis, S. R. Quake, and C. H. Yang, Nature (London) 442, 381 (2006).

${ }^{4}$ C. Monat, P. Domachuk, and B. J. Eggleton, Nat. Photonics 1, 106 (2007).

${ }^{5}$ M. Gersborg-Hansen and A. Kristensen, Appl. Phys. Lett. 89, 103518 (2006).

${ }^{6}$ M. Gersborg-Hansen and A. Kristensen, Opt. Express 15, 137 (2007).

${ }^{7}$ S. Balslev, A. M. Jorgensen, B. Bilenberg, K. B. Mogensen, D. Snakenborg, O. Geschke, J. P. Kutter, and A. Kristensen, Lab Chip 6, 213 (2006).

${ }^{8}$ K. Sakoda, Optical Properties of Photonic Crystals (Springer, Berlin, 2001).

${ }^{9}$ N. A. Mortensen, S. Xiao, and J. Pedersen, Microfluid. Nanofluid. 3 (2007).

${ }^{10}$ M. R. Lee and P. M. Fauchet, Opt. Express 15, 4530 (2007).

${ }^{11}$ N. Skivesen, A. Têtu, M. Kristensen, J. Kjems, L. H. Frandsen, and P. I. Borel, Opt. Express 15, 3169 (2007).

${ }^{12}$ L. Diehl, B. G. Lee, P. Behroozi, M. Lončar, M. Belkin, F. Capasso, T. Aellen, D. Hofstetter, M. Beck, and J. Faist, Opt. Express 14, 11660 (2006).

${ }^{13}$ M. Lončar, B. G. Lee, L. Diehl, M. A. Belkin, F. Capasso, M. Giovannini, J. Faist, and E. Gini, Opt. Express 15, 4499 (2007).

${ }^{14}$ D. Erickson, T. Rockwood, T. Emery, A. Scherer, and D. Psaltis, Opt. Lett. 31, 59 (2006).

${ }^{15}$ J. P. Dowling, M. Scalora, M. J. Bloemer, and C. M. Bowden, J. Appl. Phys. 75, 1896 (1994).

${ }^{16}$ N. Moll, R. F. Mahrt, C. Bauer, H. Giessen, B. Schnabel, E. B. Kley, and U. Scherf, Appl. Phys. Lett. 80, 734 (2002).

${ }^{17}$ S. Yokoyama, T. Nakahama, S. Mashiko, M. Nakao, M. Yamada, K. Nishio, and H. Masuda, Appl. Phys. Lett. 87, 191101 (2005).

${ }^{18}$ A. E. Vasdekis, G. A. Turnbull, I. D. W. Samuel, P. Andrew, and W. L. Barnes, Appl. Phys. Lett. 86, 161102 (2005).

${ }^{19}$ C. J. Choi and B. T. Cunningham, Lab Chip 6, 1373 (2006).

${ }^{20}$ Y. Chen, Z. Li, Z. Zhang, D. Psaltis, and A. Scherer, Appl. Phys. Lett. 91, 051109 (2007).

${ }^{21}$ S. G. Johnson and J. D. Joannopoulos, Opt. Express 8, 173 (2001).

${ }^{22}$ R. G. Hunsperger, Integrated Optics: Theory and Technology, 5th ed. (Springer, Berlin, 2002).

${ }^{23}$ K. Sakai, E. Miyai, T. Sakaguchi, D. Ohnishi, T. Okano, and S. Noda, IEEE J. Sel. Areas Commun. 23, 1335 (2005).

${ }^{24}$ X. Cheng and L. J. Guo, Microelectron. Eng. 71, 288 (2004).

${ }^{25}$ M. B. Christiansen, M. Schøler, S. Balslev, R. B. Nielsen, D. H. Petersen, and A. Kristensen, J. Vac. Sci. Technol. B 24, 3252 (2006).

${ }^{26}$ M. B. Christiansen, M. Schøler, and A. Kristensen, Opt. Express 15, 3931 (2007).

${ }^{27}$ M. Meier, A. Dodabalapur, J. A. Rogers, R. E. Slusher, A. Mekis, A. Timko, C. A. Murray, R. Ruel, and O. Nalamasu, J. Appl. Phys. 86, 3502 (1999). 